\title{
Turbine Airfoil Optimization Using Quasi-3D Analysis Codes
}

\author{
Sanjay Goel \\ University at Albany, State University of New York, 1400 Washington Avenue, Albany, NY 12222, USA \\ Correspondence should be addressed to Sanjay Goel, goel@albany.edu
}

Received 30 November 2008; Revised 25 May 2009; Accepted 20 August 2009

Recommended by Sanjay Mittal

\begin{abstract}
A new approach to optimize the geometry of a turbine airfoil by simultaneously designing multiple 2D sections of the airfoil is presented in this paper. The complexity of 3D geometry modeling is circumvented by generating multiple $2 \mathrm{D}$ airfoil sections and constraining their geometry in the radial direction using first- and second-order polynomials that ensure smoothness in the radial direction. The flow fields of candidate geometries obtained during optimization are evaluated using a quasi-3D, inviscid, CFD analysis code. An inviscid flow solver is used to reduce the execution time of the analysis. Multiple evaluation criteria based on the Mach number profile obtained from the analysis of each airfoil cross-section are used for computing a quality metric. A key contribution of the paper is the development of metrics that emulate the perception of the human designer in visually evaluating the Mach Number distribution. A mathematical representation of the evaluation criteria coupled with a parametric geometry generator enables the use of formal optimization techniques in the design. The proposed approach is implemented in the optimal design of a low-pressure turbine nozzle.
\end{abstract}

Copyright (C) 2009 Sanjay Goel. This is an open access article distributed under the Creative Commons Attribution License, which permits unrestricted use, distribution, and reproduction in any medium, provided the original work is properly cited.

\section{Introduction}

Turbine airfoil design has long been a domain of expert designers who use their knowledge and experience along with analysis codes to make design decisions. To increase productivity and performance companies are relying heavily on the vastly improved abilities of computers to use optimization and machine learning approaches. In the past, when the analysis codes took considerable time to execute on slow machines, designers used their domain knowledge and experience to effectively prune the search space and design comparable turbine airfoils efficiently. As the analysis code execution has become faster, the primary bottleneck in the design process has shifted from analyses to manual tasks of designers. Human designers are much better in forming a visual perspective and in decision-making abilities than computers. On the other hand, computers are much quicker in running codes, parsing results and analyzing numerical data. Hence, by capturing the decision-making ability of the designers and coupling them with the computation speed of computers, very efficient design systems can be created. This paper is focused on automation of a part of the process for aerodynamic design of turbines by automating the manual process and using optimization techniques to drive it.

The turbine aerodynamic design is a three-step process that is, pitchline analysis, throughflow analysis, and bladeto-blade analysis (see Figure 1). In the pitch line analysis, flow equations are solved at the blade pitch, and a free vortex assumption is used to get flow parameters at the hub and the tip. Using this analysis the flowpath of the turbine is optimized, and number of stages, work distribution across stages, stage reaction, and number of airfoils in each blade row are determined. In the throughflow analysis, the calculation is carried out on a series of meridional planes where the flow is assumed to be axi-symmetric and the boundary conditions of each stage are determined. The axisymmetric throughflow method allows for variation in flow parameters in the radial direction without using the freevortex assumption and accounts for interactions between multiple stages. In the blade-to-blade analysis, airfoil profiles are designed on quasi-3D surfaces using a computational fluid dynamics code. The design of airfoil profiles involves slicing the blade on quasi-3D surfaces, designing each section separately, and stacking the sections together to 


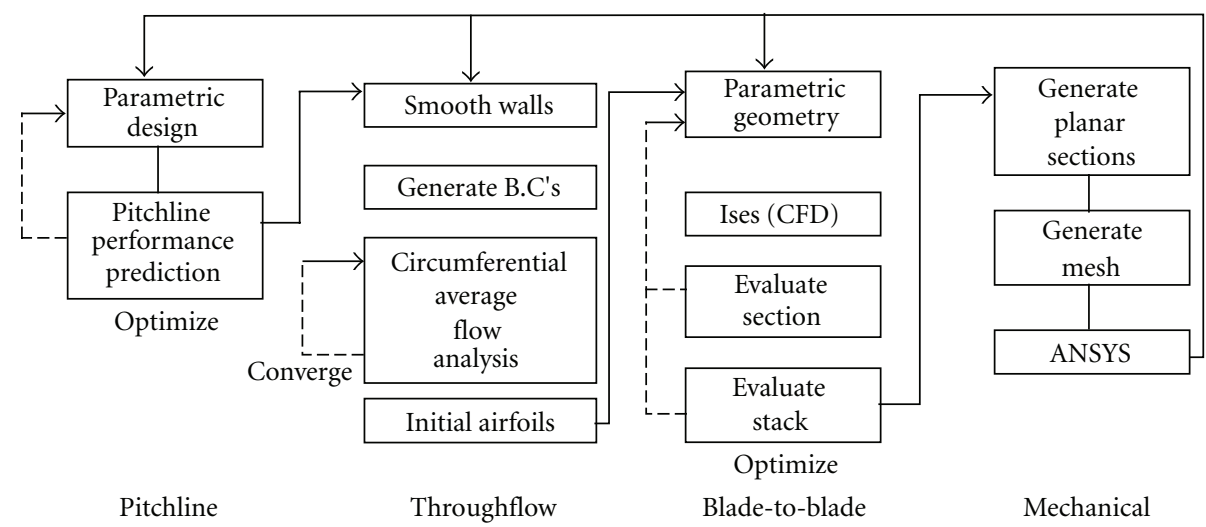

Figure 1: The turbine design process.

obtain a smooth radial geometry. The paper focuses on the automation of the design of blade profiles.

\section{Turbine Airfoil Design}

The objective of airfoil design is to define the airfoil shape so as to ensure structural integrity and minimize losses. The primary sources of losses in an airfoil are profile loss, shock loss, secondary flow loss, tip clearance loss, and end-wall loss. Profile loss is associated with boundary layer growth over the blade profile causing viscous and turbulent dissipation. This also includes loss due to boundary layer separation because of conditions such as extreme angles of incidence and high inlet Mach number. Shock losses arise due to viscous dissipation within the shock wave which results in increase in static pressure and subsequent thickening of the boundary layer, which may lead to flow separation downstream of the shock. End-wall loss is associated with boundary layer growth on the inner and outer walls on the annulus. Secondary flow losses arise from flows, which are present when a wall boundary layer is turned through an angle by an adjacent curved surface. Tip clearance loss is caused by leakage flows in the tip clearance region of the rotor blade, where the leaked flow fails to contribute to the work output and also interacts with the end-wall boundary layer $[1,2]$. The objective of the design is to create the most efficient airfoil by minimizing these losses. This often requires trading-off one loss versus another such that the overall loss is minimized.

To compute all these losses a 3D viscous analysis is required; however, due to the computational load of such a code, a quasi-3D analysis code is often used in the design process. Thus the impact of the blade geometry on $3 \mathrm{D}$ losses cannot be determined and only 2D losses can be minimized, that is, profile and shock losses. A viscous quasi-3D analysis though less computationally intense is still too expensive for use in design optimization, and an inviscid quasi-3D code is used instead. Consequently, viscous losses are not computed from the analysis code and airfoil performance is gauged by the characteristics of the Mach number distribution on the blade surface.
Several basic methods for airfoil design have been suggested in the literature, that is, inverse, direct, and adjoint. In inverse methods [3-6] for airfoil design problem, pressure distribution is prescribed on the airfoil surface and the shape of the airfoil is directly created eliminating the iterative procedure. Using inverse methods airfoil aerodynamic losses can be minimized, however, there is little control over blade geometry. Consequently, the design can end up being mechanically infeasible. Topological constraints can be included in the inverse formulation; however in that case, the desired pressure distribution may not be achievable.

In direct design methods, the airfoil design problem is posed as a constrained optimization problem where parametric geometry represents the design variables, metrics based on the Mach Number distribution comprise the objective function, and mechanical and structural requirements are imposed as constraints. Airfoil design is optimized by incrementally changing the airfoil shape and evaluating the design at each step [7-9]. This approach has been used for several applications, including, wing design [10], airfoil design [11], and supersonic planar nozzle [12] design.

Jameson [13] has proposed an aerodynamic design technique using control theory and an adjoint operator method. This is a direct design approach in which the sensitivities of the objective function to the airfoil shape are computed by solving an adjoint formulation of the flow equations where the sensitivities can be obtained in a single solution of the flow equations. It has been used for applications such as wing design [14] shape optimization of a planar diffuser [15] and aerodynamic optimization [16]. This approach requires changes to the Navier-Stokes solver, and each time a change is made to the optimization, problem the formulation needs to be modified. This approach is nonintuitive to the designers since the mesh representing the airfoil geometry needs to be changed instead of the parametric definition of the airfoil geometry that the designers are familiar with.

The most practical formulation for low-speed turbine airfoil designs still remains the direct optimization formulation based on 2D inviscid blade-to-blade solvers. This work automates the direct design process as described in the next section. 


\section{Automation of the Airfoil Design Process}

Airfoil design typically starts with an initial design that is iteratively changed until a satisfactory geometry is obtained. There are four distinct steps in the process: (1) creating initial airfoil geometry, (2) analyzing the airfoil design using CFD analysis, (3) computing airfoil quality by analyzing the Mach Number distribution, and (4) making changes to the airfoil geometry based on previous Mach Number distribution. To automate the process each step needs to be emulated computationally.

3.1. Parametric Representation. The parametric representations of the airfoils used in this work are based on the standard design tools and practices used at General Electric. There are separate models for the high-pressure and lowpressure turbine blades. The high-pressure turbine blades are subject to very high temperatures and need to be cooled. As a result, these airfoils are made thick to accommodate cooling passages inside the blades. For such thick airfoils, suction and pressure surfaces need to be manipulated independently of each other. So the airfoil is represented as a combination of two separate curves, one for the pressure side and the other for the suction side (see Figure 2). Bezier curves are well suited for these airfoils $[17,18]$. Low-pressure airfoils have lower thermal stresses, are much longer, and have a lower speed of rotation compared to high-pressure airfoils. These airfoils are usually very thin and the two-surface model does not work very well as it is very difficult to vary the pressure and suction surfaces independently and still maintain a smooth thickness distribution. For such airfoils, a meanline and thickness representation is used [19] in which a thickness distribution is superimposed on the meanline of the airfoil as shown in Figure 3. In this representation, the meanline and the thickness distribution can be varied independently, and good control of the thickness distribution is obtained. This study discusses optimization of low-pressure turbine blades. (Due to export control restrictions some details of turbine parameterization could not be discussed. In addition, due to restrictions on dissemination on company proprietary information some details of the low-pressure airfoil parameterization could not be presented.) The following parameters were used to define the airfoil geometry:

(1) stagger: angle of line joining leading \& trailing edge of the airfoil to axial,

(2) tmaxx: maximum thickness of the airfoil,

(3) c1: point of maximum thickness,

(4) c2: trailing edge included angle,

(5) c3: curvature of meanline,

(6) ratu: curvature near leading edge on upper surface,

(7) ratl: curvature near leading edge on lower surface,

(8) pcttle: incidence angle,

(9) ti: leading edge bluntness,

(10) e: ellipse ratio of the approximate ellipse fitted in the nose.

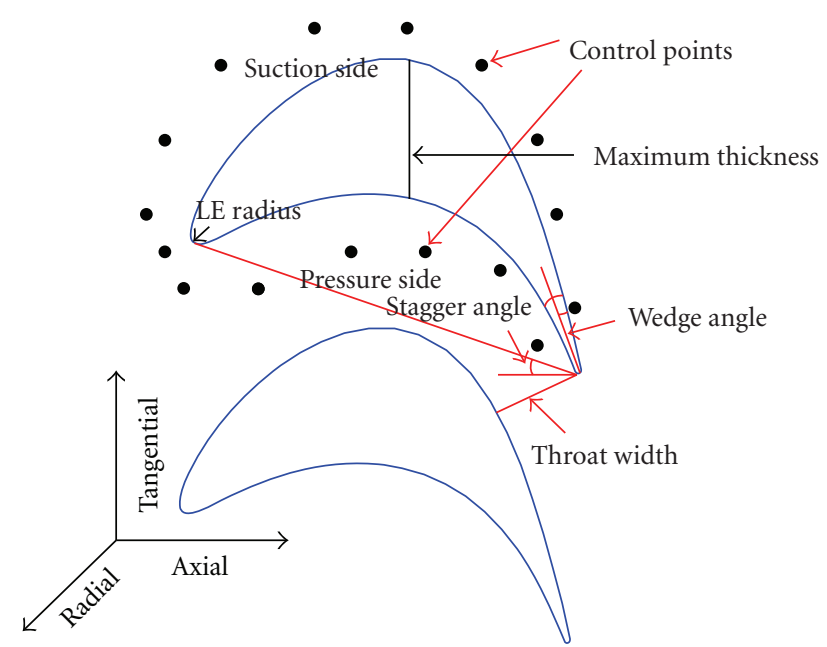

Figure 2: Parametric representation of a high-pressure airfoil section.

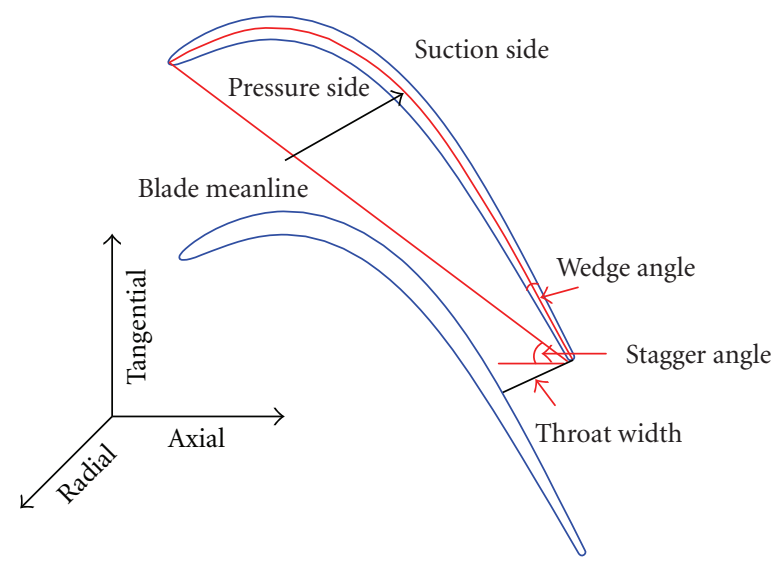

Figure 3: Parametric representation of a low-pressure airfoil section.

3.2. Quasi-3D CFD Analysis and Evaluation Metrics. A computational fluid dynamics code Ises $[3,4]$ is used in the current investigation to analyze the flow on the airfoil. Ises is an isentropic code that uses the streamline curvature method that computes the Mach Number/Pressure distribution on the airfoil surface. A major contribution of the paper is in creating numerical evaluation metrics corresponding to the visual evaluation of the designers. In the absence of a viscous code, designers usually estimate the quality of the airfoil by visually examining the Mach number distribution (Figure 4) obtained from an inviscid quasi-3D CFD solution. Since optimization techniques are driven by a numerical value of the objective function, and the visual perspective of the designer is the only proven metric available, it must be captured in a suitable numerical algorithm to provide a measure of quality of an airfoil.

3.2.1. Mach Number Evaluation. Designers use multiple criteria to evaluate a design and use their judgment in 


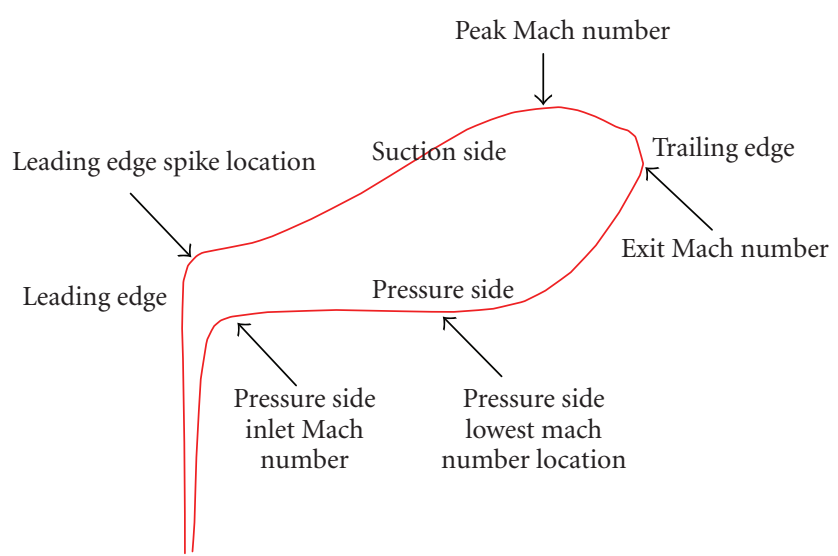

FIgUre 4: A sample Mach number distribution.

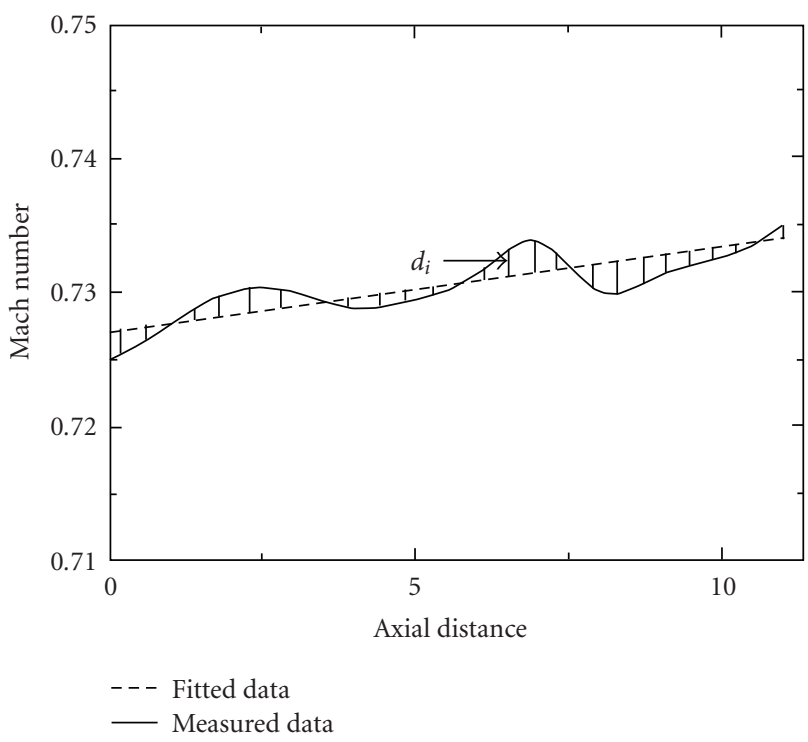

FIGURE 5: Hypothetical Mach number distribution and a first-order fit of the data.

determining the relative weight of the different criteria. Pattern recognition [20], curve fitting [8,9], classifier systems [21], neural networks [22], and Fourier analysis [23] have been used to characterize geometric shapes. The current work employs curve fitting coupled with design heuristics to compute quality metrics from the Mach number distribution and the airfoil geometry. These metrics are weighted for different designs based on individual designer preferences. Primary evaluation metrics that have been defined are diffusion, deviation, incidence deviation, and leading edge crossover. A physical interpretation of these metrics is presented below.

Diffusion is defined as the deceleration of the flow along the blade surface. It is measured as the cumulative aggregate of all flow diffusions at each point along the airfoil surface. As the flow diffuses, the boundary layer thickens, and the momentum loss in the boundary layer increases. In this case, the increased drag causes a significant loss of momentum; flow separation may result, causing much larger losses. Thus, the objective of the design is to minimize the diffusion effect. Since the impact of diffusion on the pressure and suction sides is different, separate terms are defined for the suction and pressure sides. A mathematical definition of diffusion follows:

$$
\begin{aligned}
\text { Diffusion } & =\sum_{i=0}^{n-1} d_{i}, \\
\text { where } d_{i} & =\left[\begin{array}{cc}
\left|y_{i+1}-y_{i}\right| & \text { if }\left(y_{i}>y_{i+1}\right) \\
0 & \text { if }\left(y_{i} \leq y_{i+1}\right)
\end{array}\right],
\end{aligned}
$$

where $n$ is the number of data points and $y_{i}$ is the value of the Mach number at point $i$.

Deviation is a measure of uniformity of flow acceleration along the blade surface. It is defined as the root mean square error between the Mach Number distribution on the airfoil surface and a polynomial fit of the data. Even if the flow is constantly accelerating, the rate of acceleration can have an impact on the thickening of the boundary layer causing a larger momentum loss in the boundary layer. The objective is to obtain as uniform acceleration as possible. Since the effect of nonuniform acceleration can be different on the pressure and suction surfaces, separate deviation terms are defined for the two surfaces. Figure 5 shows a hypothetical Mach number distribution on a turbine airfoil, in which the $x$-axis represents the axial distance along the turbine airfoil with an arbitrary reference location and the $y$-axis represents the corresponding Mach number values. The solid line on the plot shows the actual Mach number distribution and the dashed line shows a firs-order fit of the data; $d_{i}$ represents the difference between the actual and fitted data at different axial locations along the $x$-axis. An expression for deviation follows:

$$
\text { Deviation }=\sqrt{\left[\sum_{i=0}^{n} d_{i}^{2}\right]} \text {, }
$$

where $n$ is the number of data points and $d_{i}$ is the difference between the actual and fitted data

Incidence deviation is defined as the root mean square error between the Mach Number distribution at the nose and a polynomial fit of the data. It is a measure of the distortion of the Mach Number at the leading edge caused by a poor incidence angle of the blade. Mach Number gradients are very high at the leading edge of the airfoil resulting in extreme sensitivity to the leading edge shape necessitating a separate metric. Parameters defining the nose geometry primarily drive the incidence deviation metric during optimization. Figure 6 shows the Mach number distribution at the leading edge of a low-pressure airfoil in which, the $x$-axis represents distance along the axis of the turbine with a reference point upstream of this airfoil and the $y$-axis represents the corresponding Mach number values. The solid line on the graph shows the actual Mach number distribution and the dashed line shows a second-order fit of 


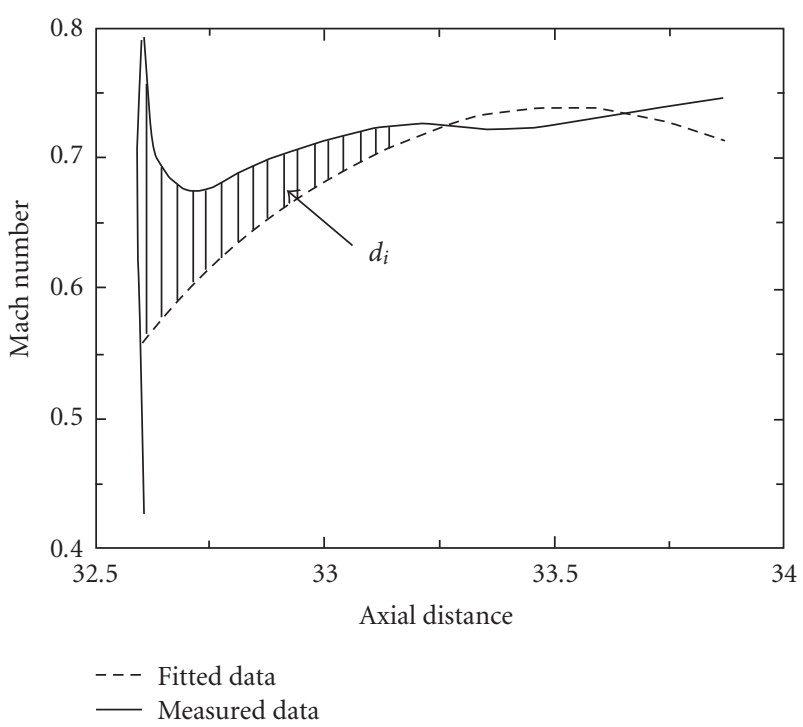

FIGURE 6: Mach number distribution at the leading edge of a blade and a 2 nd-order fit of the data.

the data; $d_{i}$ represents the difference between the actual and fitted data at different axial locations in the nose region:

$$
\text { Incidence Deviation }=\sqrt{\left[\sum_{i=0}^{n} d_{i}^{2}\right]},
$$

where $n$ is the number of data points at the leading edge and $d$ is the difference between the actual and fitted data.

Leading edge crossover is a measure of the adverse pressure gradient at the leading edge of the airfoil. Due to the boundary conditions and the shape of the airfoil, the suction side pressure may become higher than that on the pressure side close to the leading edge. This results in an increase in the loading on the rest of the airfoil, an increased risk of flow separation, and additional mechanical stresses in the airfoil. The leading edge crossover metric is computed by finding the points of intersection of the pressure and suction side Mach number distributions of the airfoil and computing the areas of the regions where the pressure side has a lower pressure than the suction side. Figure 7 shows the Mach number distribution of a low-pressure turbine airfoil in which the $x$-axis represents the distance along the axis of the turbine with a reference point upstream of the airfoil and the $y$-axis represents the Mach number values. The shaded area between the pressure and suction side Mach number distributions represents the region where the suction side Mach number is lower than that on the pressure side. The ratio of this shaded area to the total area is used to compute this leading edge crossover. The areas are computed using vector graphics:

$$
\text { LeCrossover }=\frac{A_{s}}{A_{t}},
$$

Where $A_{s}$ is the crossover area (shaded) in the Mach Number distribution and $A_{t}$ is the total area of the Mach Number distribution.

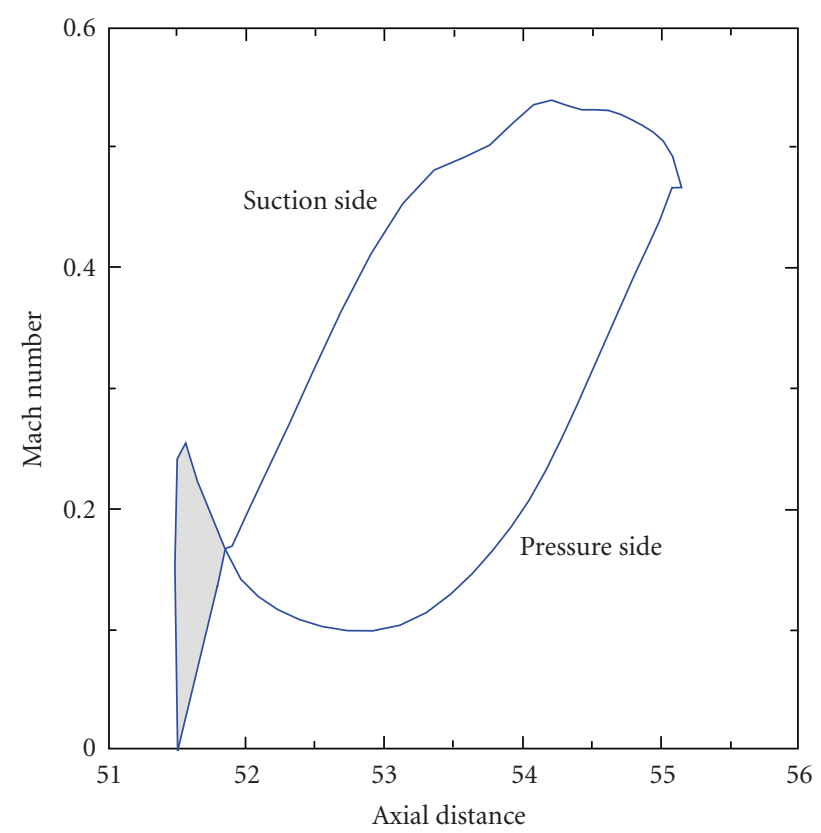

$\square$ Adverse

$\square$ Normal

FIGURE 7: Mach number distribution showing the intersection of the suction and pressure side Mach number distributions at the leading edge.

A weighted sum of the above metrics is used to define the objective function. In addition to the above metrics, diffusion on the unguided portion of the suction side is controlled by imposing constraints that are described in the next section.

3.2.2. Constraints. Constraints are imposed on the airfoil geometry to ensure that the airfoil is manufacturable and structurally feasible as well as for ensuring high aerodynamic efficiency. The structural and manufacturing constraints are based on the airfoil geometry and the aerodynamic constraints are derived from the Mach Number distribution on the airfoil. There aerodynamic constraints are defined, that is, peak-exit-ratio, peak-location, and inlet-valley-ratio. These are listed below and can be interpreted from the Mach Number distribution shown in Figure 4.

Peak-exit-ratio is defined as the ratio of the peak Mach number on the suction surface to the Mach number at the trailing edge of the blade. This is a measure of flow acceleration on the unguided portion of the airfoil (between the throat and the trailing edge). A very high turning on the unguided portion of the airfoil can lead to separation of flow or the formation of a shock on the trailing edge. By putting a constraint on the maximum peak-exit-ratio, chances of separation are minimized.

Peak-location is the normalized location of the peak Mach number on the suction side. It is desirable to have an increasing Mach number as far along on the suction side as possible to prevent a thickening of the boundary layer. 
Imposing a constraint which allows the peak to occur after $65 \%$ of the blade width guards against upstream diffusion and helps in achieving a smooth accelerating Mach number on the suction side.

Inlet-valley-ratio is the ratio of the Mach number at the inlet of the airfoil on the pressure side, to the minimum Mach number on the pressure side. This constraint controls the diffusion near the inlet on the pressure side and restricts the thickening of the boundary layer, reducing chances of flow separation. Sample constraints are described below

(i) $0<$ peak_exit $<1.3$ (Peak to exit ratio),

(ii) $0.6<$ peak_exit_loc $<1$ (Location of peak Mach Number along the chord),

(iii) $0<$ peak_imin $<1.5$ (Ratio of inlet to minimum Mach Number on pressure side).

Constraints are also imposed on (1) the curvature change on the unguided portion of the airfoil (unguided turning) (2) the difference between the blade meanline angle and the flow angle at the trailing edge (over turning), and (3) the difference between the inlet angle and the metal angle at the inlet (delta1). These additional checks further ensure that the designed airfoil stays within design practice guidelines.

To ensure mechanical and structural feasibility, constraints are imposed on the blade geometry. The primary geometry parameters are cross section area, maximum thickness of airfoil, wedge angle, and nose radius. In cooled airfoils, the constraints on the geometry stem from the necessity to construct cooling channels in the airfoil; these constraints are dictated by manufacturing requirements. In low-pressure airfoils, these constraints are primarily driven by stresses and manufacturing limitations. Most of these constraints have soft limits on them; that is, it is best to have the responses within a given range, beyond a threshold of the range a penalty that increases nonlinearly with increased violation of the constraint is added to the objective function. The geometry-based constraints used in the problem formulation are listed below:

(i) $0<$ carea $<10$ (Airfoil Cross Section Area),

(ii) $.035<$ rad_le $<5$ (Leading Edge Radius),

(iii) $2<$ wedge < 6.6 (Wedge Angle),

(iv) $0<$ te $<30$ (Trailing Edge Thickness),

(v) $-1<$ ovt $<4$ (Over Turning),

(vi) $-30<$ delta $1<30$ (Difference between blade Incidence angle and flow angle),

(vii) $0.04<$ tmax_c $<0.2$ (Maximum thickness to chord ratio),

(viii) $0<p c t z<2$ (Normalized location of max. airfoil thickness along chord).

3.2.3. Stack Constraints. The design for $3 \mathrm{D}$ airfoils was done on multiple 2D planes, which were stacked together and the full 3D geometry was obtained by interpolating between the sections. In order to maintain a smooth geometry across multiple sections, geometry continuity must be maintained from section to section. During the manual design process, designers change multiple sections simultaneously and mentally keep track of the relative shapes of the sections while changing them. Ensuring smoothness is often an iterative process; once the individual airfoil sections are optimized, the sections are assembled into a stack with polynomial fits across the sections. The sections are then tweaked to obtain radial smoothness. The manual design process often alternates between smoothing the airfoil stack and optimizing the airfoil sections. In an automated design system, geometry constraints between adjacent sections can be enforced via curve fitting. Coefficients of the fit can be varied instead of the geometry parameters of individual airfoils.

\section{Penalty Functions}

The starting design for the optimization of the airfoil aerodynamic design problem is usually infeasible and the problem is sometimes over constrained. A lot of the constraints are formulated based on the prior experience of the designers and are fairly conservative. Constraint boundaries, however, are flexible and can be changed at the designer discretion. A constraint is relaxed if it results in a significant reduction in the violation of some other constraint or in the improvement of turbine performance. For some constraints even when the response quantity is within bounds, the designers prefer to keep the value of the response quantity either as low as possible (within bounds), as high as possible (within bounds), or at the midpoint of the constraint bounds to achieve greater reliability, performance, or cost advantage. The design process is thus a complex sequence of tradeoffs with soft constraint boundaries.

To simulate this behavior during optimization a set of Penalty Functions are used in the formulation. These functions vary linearly within the constraint bounds and exponentially outside the bounds. Imposing such penalty functions on the response quantity ensures that the response quantity values remain in close proximity to their allowable limits, while allowing sufficient flexibility in traversal of the search space during optimization. The basic form of the penalty function used for incorporating constraints into the objective function in the optimization formulation is shown below:

$$
\operatorname{Penalty}\left(x_{t}\right) P=s \times\left|x_{t}\right|+\beta \times\left|\frac{x_{t}}{\gamma}\right|^{\alpha} \text {, }
$$

where $x_{t}$ is the value of the response quantity, $\gamma$ is the upper limit of the allowable range, $\alpha$ controls the deviation of the function from a linear behavior when the response quantity value lies outside the allowable limits, $\beta$ controls the deviation from the linear when the response quantity value is within allowable limits, and $s$ is the rate of change when response quantity value is within allowable limits.

The allowable limits and the response quantities are transformed such that they are symmetric about the mid 
point of the constraint bounds (we will call it the origin). This transformation is shown in (6):

$$
\left[\begin{array}{c}
\text { mean }=\frac{\mathrm{lb}+\mathrm{ub}}{2.0} \\
y=\frac{\mathrm{ub}-\mathrm{lb}}{2} \\
x_{t}=x-\text { mean }
\end{array}\right],
$$

where $\mathrm{ub}$ is the upper bound of the response quantity, $\mathrm{lb}$ is the lower bound of the response quantity, $x$ is the actual value of the response quantity, $x_{t}$ is the transformed value of the response quantity, and $-\mathrm{r}$ to $+\mathrm{r}$ is the feasible range for $x_{t}$.

This penalty function defined in (5) is symmetric about the midpoint of the constraint range. It evaluates to a value of zero when $x_{t}$ is at the midpoint of the allowable range. The penalty increases as $x_{t}$ moves away from the mid point in either positive or negative direction. When the response quantity is within bounds, the linear part of the penalty function dominates, and when the response quantity is outside the bounds, the nonlinear part of the penalty function dominates. The effect of the nonlinear portion of the penalty function is very small when the value is within allowable limits, since the value of $\beta$ is very small, $\alpha$ is greater than 3 , and the exponent term is less than $1\left(x_{t} / \gamma \leq\right.$ $1, \alpha \geq 3.0$ ) but it increases rapidly as the constraint violation increases. Typical values used in the formulation are $\beta=$ $0.05, \alpha=3-5$, and $s=1$. The value of the penalty function thus increases monotonically on either side of the origin. It varies linearly while the value of $x_{t}$ is within the allowable range and becomes exponential as it moves beyond the constraint boundary.

To obtain a monotonically increasing penalty function a variation of the penalty function shown in (5) is used. In this penalty function (shown in (7)) the absolute value restriction is removed from the penalty function for both the linear and nonlinear terms, the value of the slope $s$ is positive, and the value of $\alpha$ is set to an even integer. When the value of $x_{t}$ is negative, the linear portion of the penalty function is negative, and as the value of $x_{t}$ drops further the penalty function value also drops (more negative). When $x_{t}$ is zero, the penalty function is also zero. When $x_{t}$ is positive, the penalty function is positive and it becomes higher as the value of $x_{t}$ increases. The penalty function is thus monotonically increasing from a negative value to a positive value as the value of $x_{t}$ increases from the lower bound to the upper bound. The penalty function is positive (exponent of the nonlinear term is an even integer) and rises very rapidly as the constraint violation increases when a constraint is violated at either constraint boundary (since the linear term is insignificant and the nonlinear term becomes dominant):

$$
\operatorname{Penalty}\left(x_{t}\right)=s \times x_{t}+\beta \times\left(\frac{x_{t}}{\gamma}\right)^{\alpha} .
$$

Similarly to get a monotonically decreasing function, (7) is used where the absolute value restriction is dropped, the value of $\alpha$ is positive and the value of the slope, and $s$ is negative. When the response quantity value $x_{t}$ is negative and the linear part of the penalty function is positive, it becomes zero when $x_{t}$ is zero and becomes negative when $x_{t}$ is positive. Since the linear part dominates the nonlinear part of the penalty function, the penalty function monotonically increases from a positive value to a negative value as the value of $x_{t}$ varies within the constraint bounds from $-r$ to $+r$. When the constraint is violated at either of the bounds, the nonlinear part dominates the penalty function. The exponential term is positive because the exponent of the nonlinear part of the function is even.

Two different penalty functions defined above were used in the turbine design problem formulation. The first function uses (5) in which the slope $s$ is set to zero such that the value of the penalty function is zero (nonlinear term is negligible) when the response quantity value is within bounds. When the response quantity value is out of bounds, the value of the nonlinear part of the penalty function becomes dominant and penalizes a violation of the constraint. The second function uses (6) to generate a monotonically increasing function by setting the value of $s$ positive. Thus the constraint will try to keep the value of the response quantity $x_{t}$ low even when $x_{t}$ is within bounds and will keep it close to its bounds even when it exceeds its bounds in either direction.

4.1. Problem Formulation. The objective function includes the performance metrics and constraints where the violations are included via penalty functions. Two different types of penalty terms, $P_{c 1}$ and $P_{c 2}$, are used. In $P_{c 1}$, the penalty increases linearly as the value increases within bounds; however, it increases nonlinearly if either the lower or the upper bound is violated. In $P_{c 2}$ there is no contribution of the penalty term to the objective function if the parameter is within bounds; however, the penalty increases nonlinearly if the constraint is violated from either bound. Three different functions are used for capturing the variations within the bounds depending on whether the midpoint or one of the bounds is the preferred value of the constraint variable. The penalty terms $P_{c 1}$ and $P_{c 2}$ are applied to the parameters shown within the parenthesis in the following equation:

Minimize. Wsdiff $*$ Suction_Diffusion + Wpdiff $*$ Pressure_Diffusion + Wsdev $*$ Suction_Deviation + Wpdev $*$ Pressure_Deviation + Wledev $*$ Le_Deviation + Wcross $*$ Le_Crossover $+P_{c 1}$ (peak_exit, peak_exit_loc, peak_imin, carea $)+P_{c 2}$ (rad_le, wedge, te, ovt, staggr, delta1, tmax, tmax_c, pctz).

Here W's are the weighting coefficients associated with the fitness measures. These factors can vary for different problems based on the requirements of the specific problem. The design variables and typical range of variations are listed in Table 1 and the constraints imposed on the problem are listed in Table 2.

During the design of an airfoil, multiple sections are designed concurrently, and the objective function is a sum of the objective functions of all the cross sections being designed. Constraints for all the sections are also included in the problem formulation. Polynomial fits are used to represent the radial variation of the design variables; thus the 
TABLE 1: Design variables used in the formulation of the airfoil design problem.

\begin{tabular}{lcc}
\hline Design variable & Lower bound & Upper bound \\
\hline c1 & 0.2 & 0.5 \\
c2 & 0.25 & 0.75 \\
c3 & 0.25 & 0.75 \\
Tmaxx & 0.05 & 0.15 \\
Staggr & 8 & 40 \\
Pcttle & -0.25 & 0.25 \\
Ratl & 0 & 4 \\
Ratu & 0 & 4 \\
Ti & 0 & 1 \\
E & 1 & 5 \\
\hline
\end{tabular}

TABLE 2: Constrained variables used in the formulation of the airfoil design problem.

\begin{tabular}{lcc}
\hline Constrained variable & Lower bound & Upper bound \\
\hline peak_exit & 0 & 1.3 \\
peak_exit_loc & 0.6 & 1 \\
peak_imin & 0 & 1.5 \\
Carea & 0 & 10 \\
rad_le & 0.035 & 5 \\
Wedge & 2 & 6.6 \\
Te & 0 & 30 \\
Ovt & -1 & 4 \\
deltal & -30 & 30 \\
tmax_c & 0.04 & 0.2 \\
Pctz & 0 & 2 \\
\hline
\end{tabular}

objective function becomes a combination of the coefficients of the fits across multiple sections rather than individual parameters for each section. A second-order polynomial fit is used in the formulation; so corresponding to each metric we have three coefficients.

The solution to the problem can be attempted using a variety of optimization techniques including numerical optimization, genetic algorithms, simulated annealing, and heuristic search. In the current investigation, the BFGS variable metric method implemented in an optimization code ADS was used. A one-dimensional search technique was used in which the search was bounded followed by use of polynomial interpolation.

\section{Results}

In the test case presented here, a low-pressure turbine nozzle is optimized. The flowpath of the low-pressure turbine used in the investigation is shown in Figure 8. The radial distances in the figure are measured with reference to the centerline of the engine and the axial distances are measured with reference to a point upstream of the first stage of the turbine. The horizontal lines in the figure represent the streamlines of the flow. Thirteen streamlines are shown, the top and bottom of which coincide with the casing and the hub,

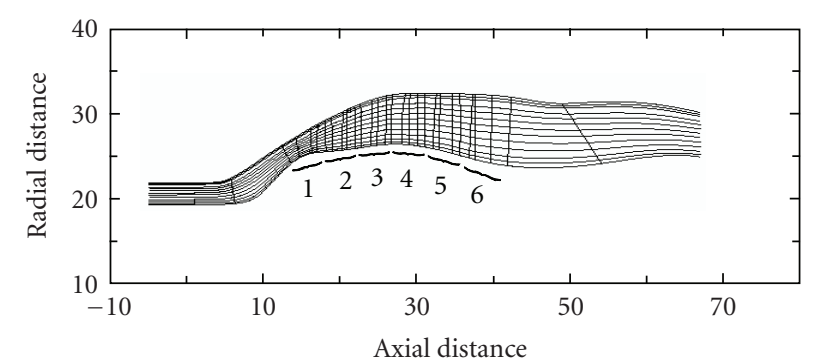

FIGURE 8: Flowpath of the turbine used in the current investigation.

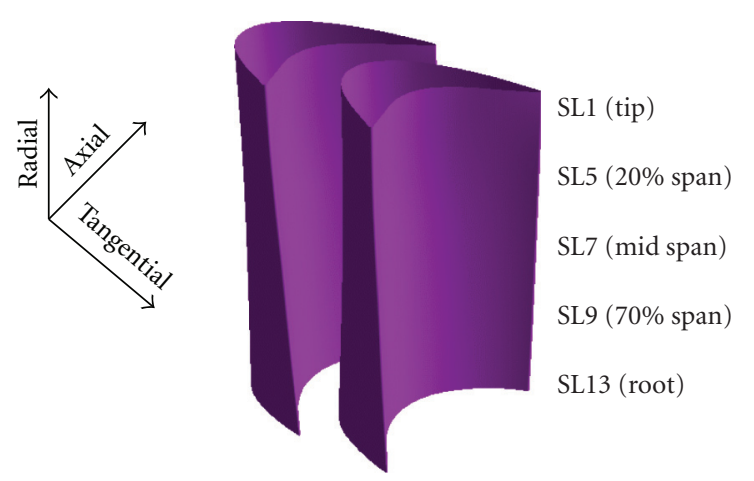

FIGURE 9: Schematic representation of an airfoil showing streamlines along the radial direction.

respectively. The vertical lines represent the edges of the blade rows and the location of the frame. The turbine has six stages, each stage composed of two blade rows. The first blade row consists of nozzles and the second blade row consists of buckets. The stages are numbered from 1 to 6 in the figure.

In the current investigation, stage 5 nozzle was designed using sections from five streamlines equally spaced along the blade span (hub to tip). Figure 9 shows the approximate locations of the streamlines for an airfoil in which the first and the last streamlines are shown at the hub and tip, respectively. In reality however streamlines at 5\% and $95 \%$ span were used instead of streamlines directly on the hub and tip because Mach number distributions very close to the end walls are distorted by the end wall effects and not representative of the flow away from the walls. The starting solution for the test case was obtained by estimating the airfoil shape based on shapes of similar airfoils designed in the past. All the Mach number and airfoil geometry plots use the same reference radial and axial locations as shown in Figure 9.

To ensure slope and curvature smoothness of the geometry, second-order polynomials were used to represent the radial distribution of geometry parameters. Thus there are three design variables for each geometry parameter, that is, $\mathrm{C}_{0}, \mathrm{C}_{1}$, and $\mathrm{C}_{2}$. These are the coefficients of the secondorder polynomial representing the geometry parameter. The coefficients of the fit match well with the starting design since the design is based on a previously designed airfoil. 


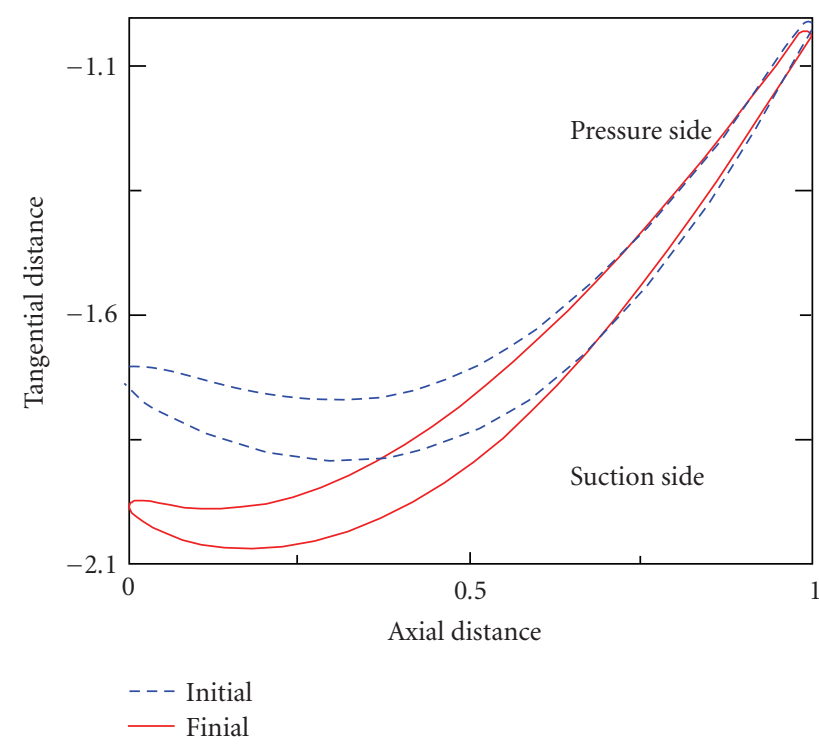

(a)

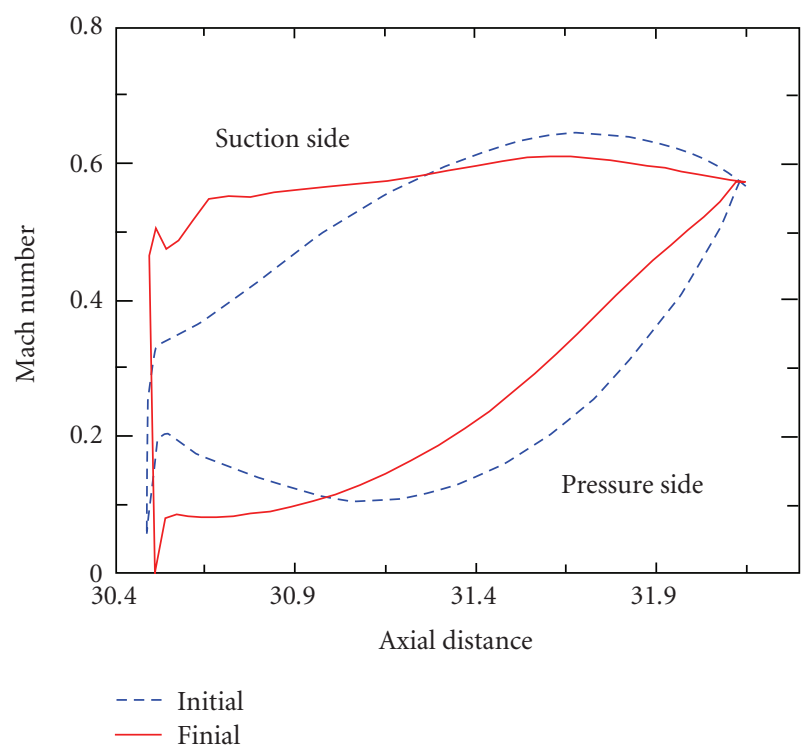

(b)

FIgURE 10: Initial and final airfoil sections and Mach Number distributions for streamline at the nozzle tip [SL 3].

Subsequently the smoothness is maintained since the parameters are not changed directly but rather the coefficients of the polynomials are varied. The geometry parameters which describe the low-pressure turbine airfoil geometry are Stagger, Tmaxx, C1, C2, C3, Ratu, Ratl, Pcttle, ti, and e; these parameters are described in Section 3.1 and some of these are graphically described in Figure 3. These geometry parameters are varied within limits typically prescribed in design practice and on the basis of prior experience and manufacturing limitations. The limits for these parameters are described along with the results for each specific test case.

Performance constraints based on design practice guidelines are shown in the list below. These are hard constraints; one or more of these are usually violated in the starting solution. Even within bounds, constraints like Peak-exitratio and Inlet-valley-ratio are included in the objective function and minimized. Detailed definition of each of these is provided earlier in Section 3.2.2:

(i) $0.0<$ Peak-exit-ratio $<1.2$,

(ii) $0.65<$ Peak-location $<1.0$,

(iii) $0<$ Inlet-valley-ratio $<2.0$.

The geometry constraints for manufacturing requirements and mechanical stress limits are rad_le, wedge, ugt, te, ovt, delta1, tmax_c, and carea. The metrics comprising the objective function are suction_diffusion, pressure_diffusion, suction_deviation, pressure_deviation, le_deviation, and le_crossover. Detailed description of each of these parameters is provided in Section 3.2.1. The weighting factors corresponding to each component are provided along with the results of the test cases.

5.1. Low-Pressure Turbine Nozzle Design. The results of the optimization for a low-pressure turbine nozzle are contained in Tables 3, 4, and 5 shown in appendix. The tables include the initial and final values of the design variables, constraints, and objective function components at the pitchline (streamline 7). The geometry and Mach number distributions of the initial and final airfoil sections for streamlines 3, 5, 7, 9, and 11 are shown in Figures 10-14. In each figure the initial and final airfoil geometry and Mach number distribution are superimposed in order to compare the two solutions easily. The Mach number distributions of the initial and final airfoils clearly show a significant change in the final design compared to the initial design. The excessive diffusion on the pressure side has been reduced significantly, and the acceleration on the suction side has become more uniform than before. Also, the loading on the leading edge has increased, making the loading distribution more uniform across the blade width. The spike in the Mach number at the leading edge of the airfoil is reduced considerably. The small discontinuities in the Mach number distribution near the leading edge are due to limitations in mathematical representation, which result in a poor resolution of the geometry in regions where the curvature changes rapidly. Note that in the nose region, the angle changes by almost 360 degrees over a very small region. These nonuniformities in the shape representation of the nose region can usually be smoothed out manually using a geometry modeler, which models the nose region with high precision independently of the rest of the airfoil. Diffusion on trailing edge of the airfoil on the suction side is also reduced, which further reduces the profile losses. Figure 15 shows the value of the overall objective function with respect to the design iterations. The entire optimization required 117 iterations and the objective function improved by 41.8943 . The design converged in about 40 iterations. The spikes in the convergence history are due to constraint violations, which cause the objective function value to increase suddenly due to the nonlinear penalty terms appended to the objective. 


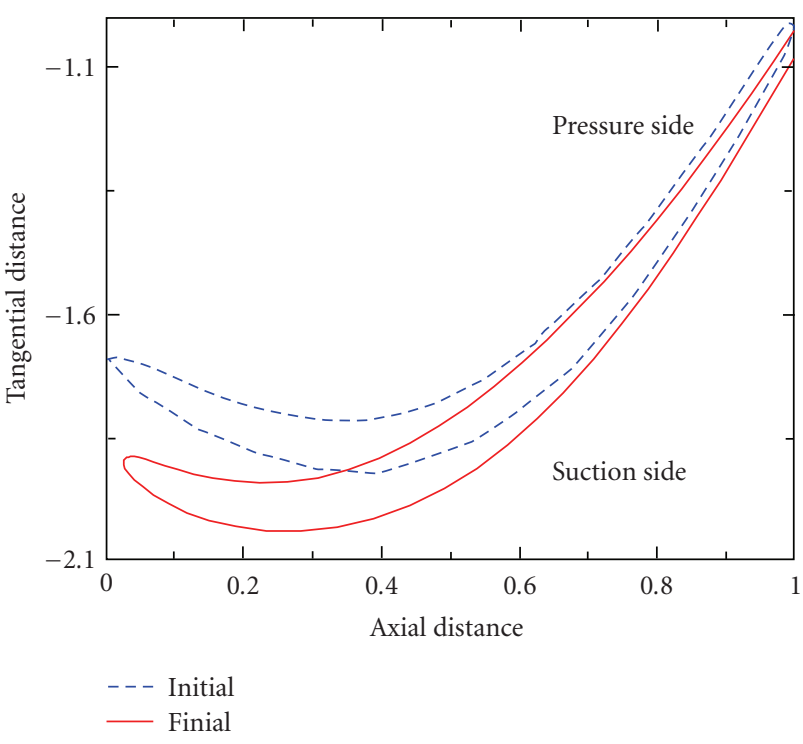

(a)

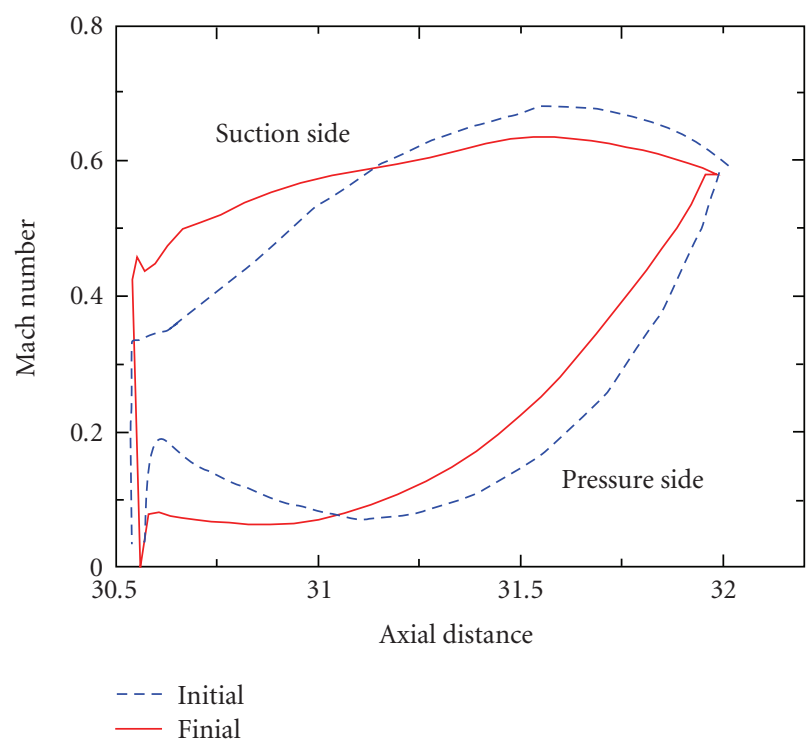

(b)

Figure 11: Initial and final airfoil sections and Mach number distributions for streamline between the nozzle tip and pitch [SL 5].

Fletcher-Reeves algorithm for unconstrained minimization with Golden Section search was also used for optimization of the nozzle. Airfoil sections very similar to those obtained in the previous example were obtained. The iteration history for the optimization is shown in Figure 16. The optimization required 113 iterations to converge and the objective function value in the final design was 33.8182. Spikes observed in the convergence history are again due to nonlinear penalty functions associated with constraint violations. As evident from Figures 15 and 16 no significant impact was observed by changing the search technique.

The optimized airfoils are more front loaded compared to the initial design which may or may not be desirable based

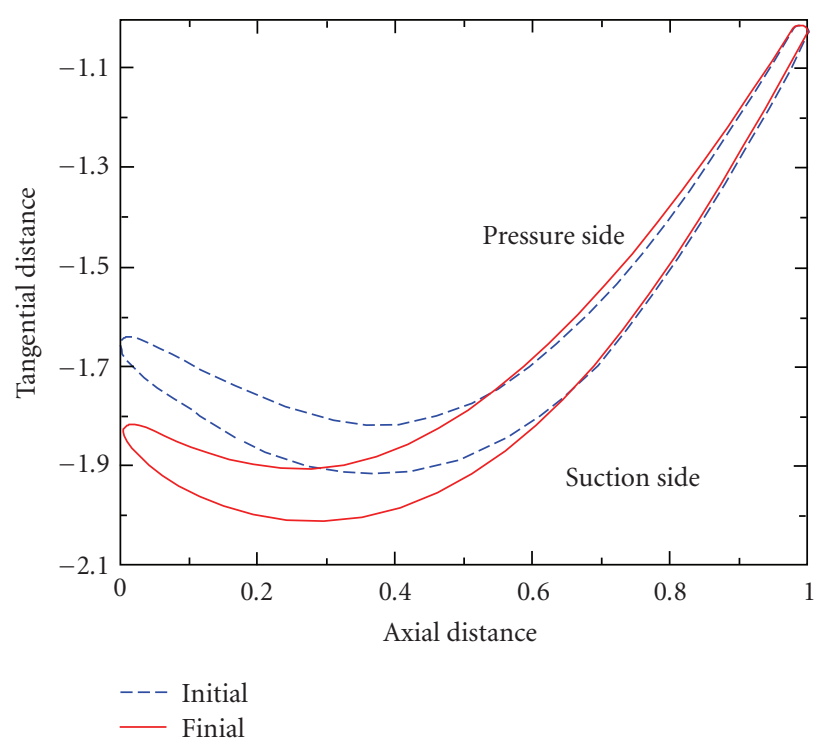

(a)

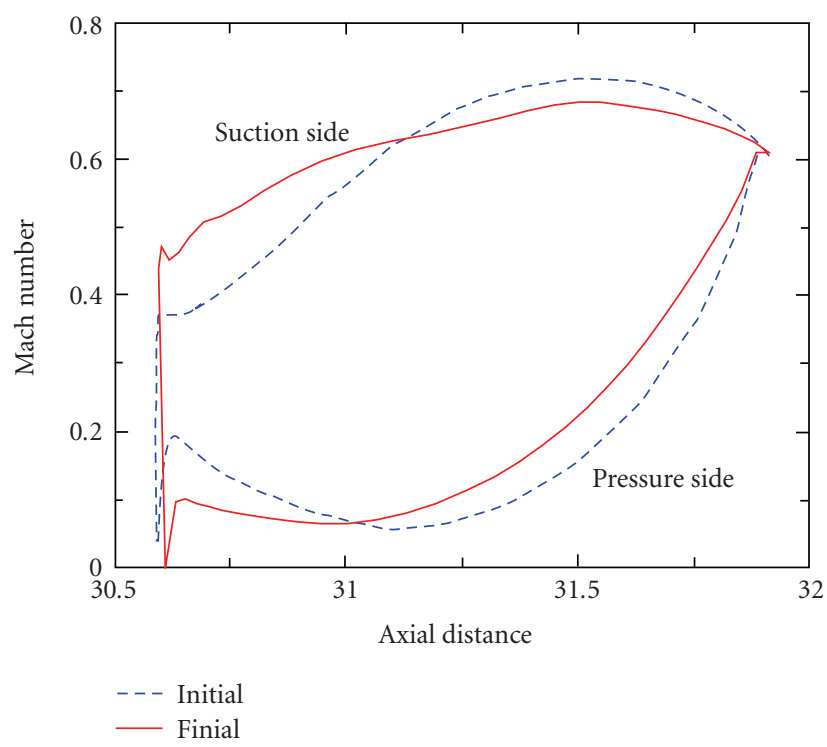

(b)

Figure 12: Initial and final airfoil sections and Mach number distributions for streamline at the nozzle pitch [SL 7].

on the designer preference. The objective function weights could be changed such that the loading on the leading edge is reduced. During the optimization, radial smoothness of the airfoil geometry is ensured by maintaining a 1st- or 2ndorder polynomial smoothness in the distribution of each design variable in the radial direction. Figure 17 shows the blade passage from the leading edge side and the airfoil surfaces; edges as well as the blade passage appears smooth and uniform.

\section{Conclusions}

The paper presents a mathematical formulation for design of turbine airfoils using 2D geometry models and 2D inviscid 


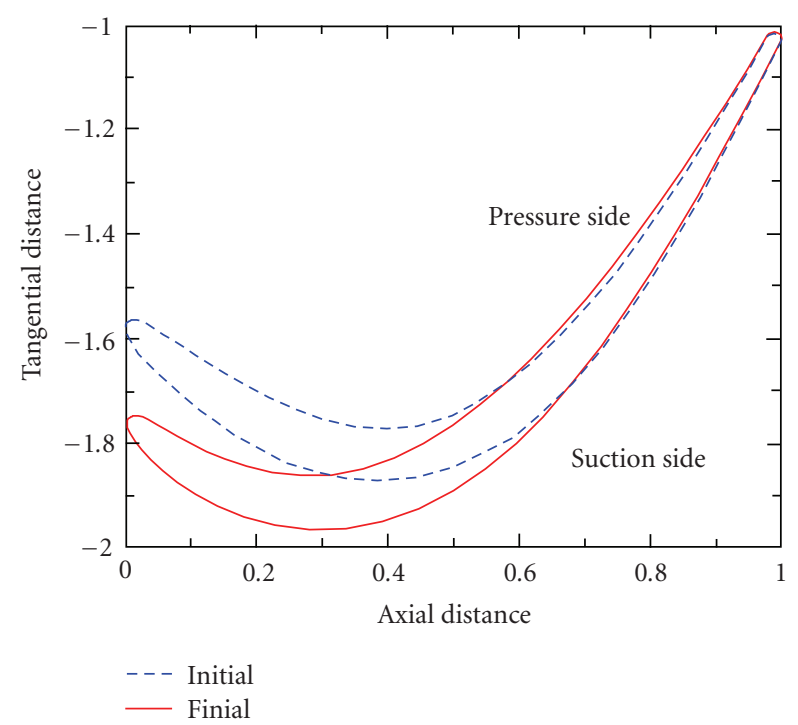

(a)

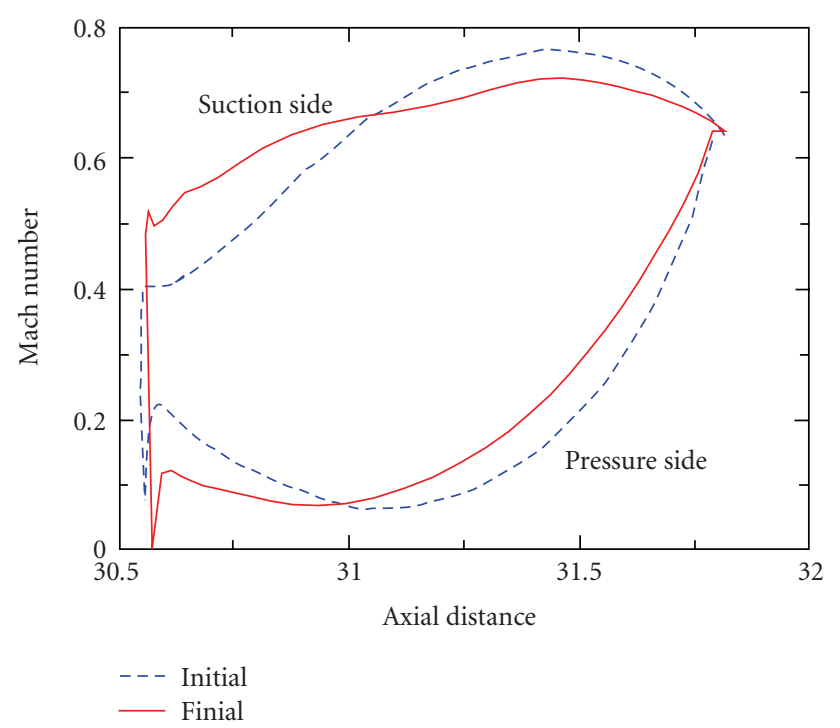

(b)

FIGURE 13: Initial and final airfoil sections and Mach Number distributions for streamline between the nozzle pitch and root [SL 9].

analysis codes. The reduced computational complexity of the new formulation compared to $3 \mathrm{D}$ viscous analysis makes the airfoil design problem amenable to the use of formal optimization methods. The paper presents results from design of a low-pressure turbine nozzle. There are three primary contributions of this work: (1) a numerical metric for emulating designer judgment in evaluation of airfoil Mach Number Distribution, (2) an optimization formulation for design of airfoil sections, and (3) a methodology which allows design of 3D airfoils by simultaneous design of multiple 2D sections. Designer heuristics are computed using curve fits and error norms. A set of penalty functions has been defined which allows for flexible constraint boundaries and influence constrained variables even within constraint limits. In the

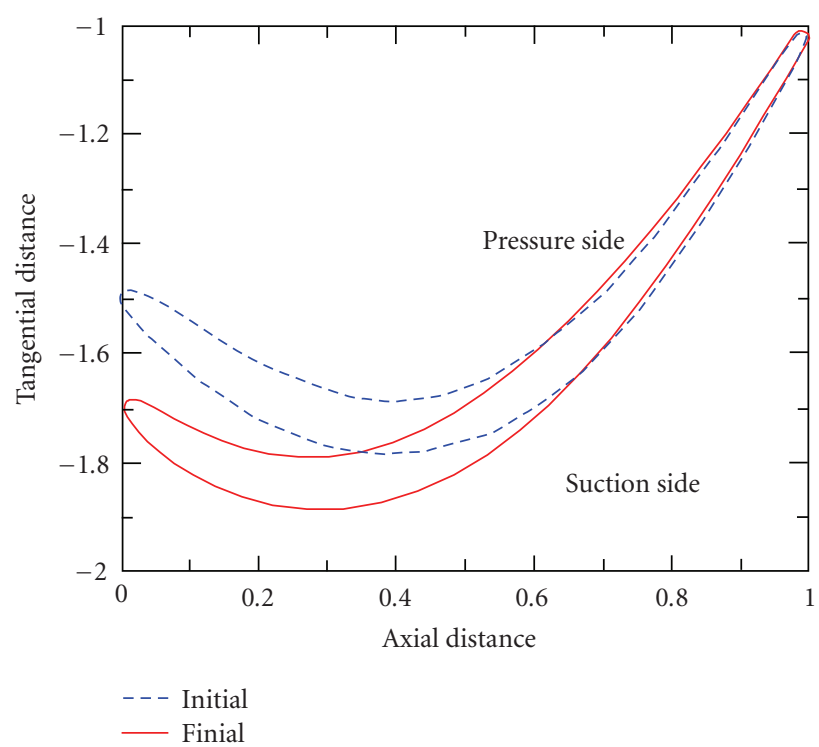

(a)

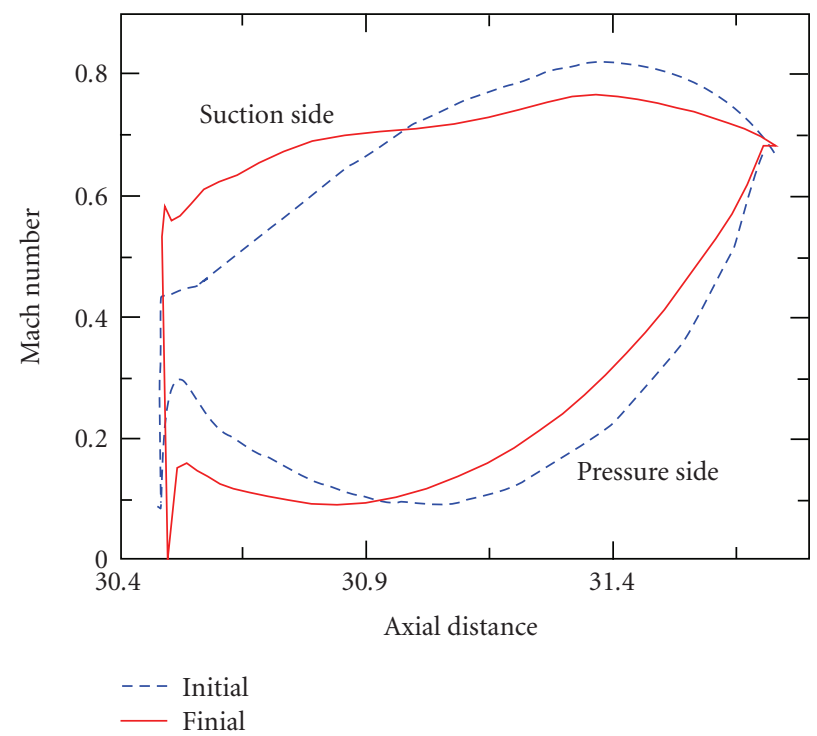

(b)

Figure 14: Initial and final airfoil sections and Mach Number distributions for streamline at the nozzle root [SL 11].

new approach multiple two-dimensional sections of the airfoil are designed with constraints on radial smoothness using polynomial fits on the parametric geometry variables in the radial direction.

Airfoil design is a labor intensive, repetitive, and cumbersome task for the designers and is a bottleneck for both the design cycle and rapid generation of inputs for complex multistage analyses. Automating the design process significantly cuts down the design cycle time and facilitates the task of running multistage analysis by rapidly generating airfoil geometries.

While designing an airfoil, it is hard to establish the existence of a unique optimum. Multiple evaluation criteria which are weighted together to define the objective function 


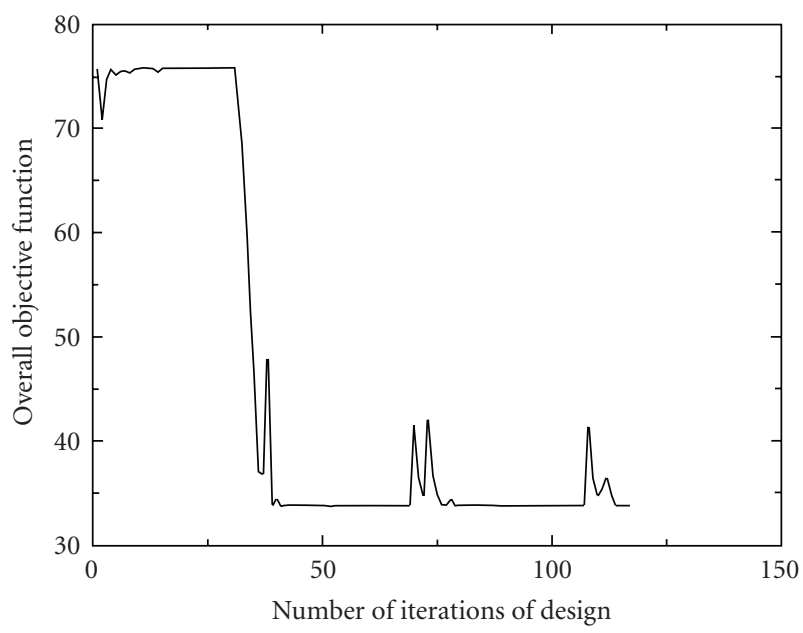

FIGURE 15: Change in objective function for low-pressure turbine nozzle optimization using gradient-based search (ADS: ISTRAT = $3, \mathrm{IOPT}=3, \mathrm{IONED}=3$ ).

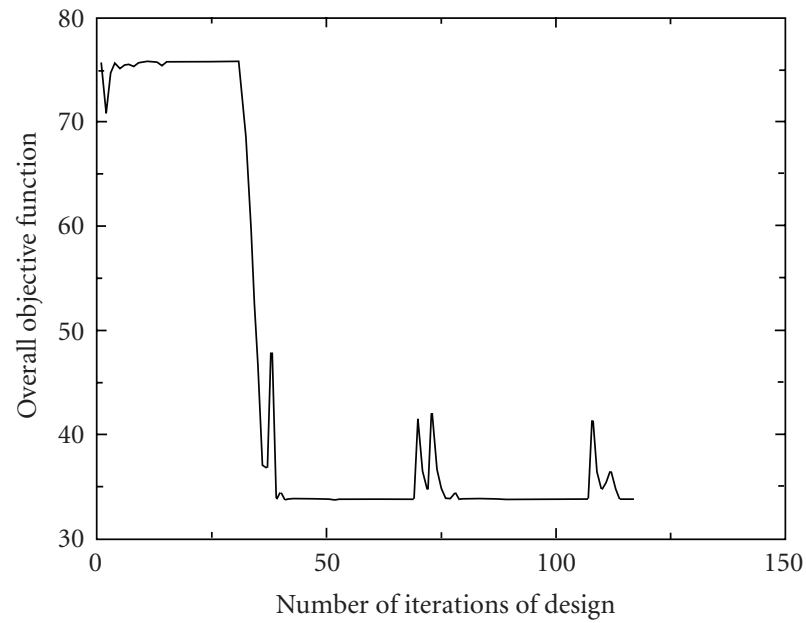

FIgURe 16: Change in objective function for low-pressure turbine nozzle optimization using gradient-based search $(\mathrm{ADS} \rightarrow$ Istrat $=$ 0 , Iopt $=1$, Ioned $=1$ ).

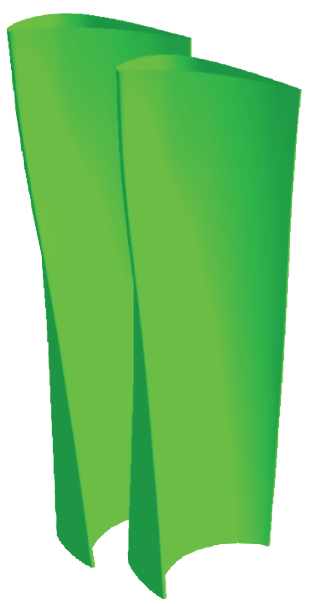

FIGURE 17: A 3D model of an airfoil showing the passage between adjacent airfoils.
TABLE 3: Design variables.

\begin{tabular}{lcccc}
\hline Name & Lower bound & Upper bound & Initial value & Final value \\
\hline peak_exit & 0 & 1.3 & 1.15239 & 1.09774 \\
peak_exit_loc & 0.6 & 1 & 0.655226 & 0.702005 \\
peak_imin & 0 & 1.5 & 3.84903 & 1.63175 \\
rad_le & 0.035 & 5 & 0.0177245 & 0.0177331 \\
Wedge & 2 & 6.6 & 2.3837 & 2.87872 \\
Ugt & 5 & 18 & 16.0004 & 12.2213 \\
Te & 0 & 30 & 0.0299102 & 0.0299102 \\
Ovt & -1 & 4 & 3.22223 & 1.77036 \\
Stagger & 0 & 50 & 30.9603 & 39.1863 \\
Deltal & -30 & 30 & -18.2188 & -18.2188 \\
Tmax & 0 & 2 & 0.141241 & 0.138897 \\
tmax_c & 0.04 & 0.2 & 0.094285 & 0.0837007 \\
Pctz & 0 & 2 & 0.389531 & 0.40175 \\
Carea & 0 & 10 & 0.165028 & 0.174538 \\
\hline
\end{tabular}

TABLE 4: Objective function components.

\begin{tabular}{lccc}
\hline Parameter & Weight & Initial value & Final value \\
\hline dev_suction & 10 & 0.177097 & 0.0644436 \\
dev_pressure & 2.5 & 0.0450137 & 0.0112053 \\
diff_suction & 5 & 0.186932 & 0.128518 \\
diff_pressure & 1.5 & 0.308536 & 0.0656558 \\
le_crossover & 50 & 0 & 0 \\
le_smoothness & 2.5 & 0.0035042 & 0.00875813 \\
peak_exit & & 1.9005 & 1.80434 \\
peak_exit_loc & & 0 & 0 \\
peak_imin & & 12.287 & 2.43818 \\
Carea & & 0.212921 & 0.225093 \\
tmax/c & & 0 & 0 \\
Wedge & & 0 & 0 \\
Ugt & & 0 & 0 \\
Ovt & & 0 & 0 \\
Objective function value & & 18.4653 & 6.53994 \\
\hline
\end{tabular}

TABLE 5: Constraint variables.

\begin{tabular}{lcccc}
\hline Name & Lower bound & Upper bound & Initial value & Final value \\
\hline c1 & 0.2 & 0.5 & 0.35 & 0.35 \\
c2 & 0.25 & 0.75 & 0.5 & 0.632109 \\
c3 & 0.25 & 0.75 & 0.5 & 0.569811 \\
Tmaxx & 0.05 & 0.15 & 0.139516 & 0.139516 \\
Stagger & 8 & 40 & 31.643 & 39.4644 \\
Pcttle & -0.25 & 0.25 & 0 & 0 \\
Ratl & 0 & 4 & 1.25 & 2.7032 \\
Ratu & 0 & 4 & 2.59442 & 2.72716 \\
Ti & 0 & 1 & 0.5 & 1 \\
E & 1 & 5 & 3 & 2.04457 \\
\hline
\end{tabular}


and the relative importance of these are determined based on designer experience. Furthermore, the analysis codes are not exact, and even with precisely defined quality metrics, a significant margin of error remains. In manual design the evaluation criteria are implicitly considered by the designer, with weighting factors based on past experience and individual biases. Subjectivity is introduced into the design process since the evaluation criteria for the design are partially based on heuristics abstracted from designer experiences. Thus in order to completely understand the results of airfoil optimization, an evaluation of the qualitative changes to the design is essential after the optimization is completed. Over time as the metrics to evaluate airfoil design become more acceptable, a standard metric will emerge, till such time designers will need to tinker with the weights to suit their own preferences.

\section{Appendix}

This appendix presents the initial and final design configuration for the low-pressure turbine airfoil during optimization. The values are presented at the pitchline (approx. axial midpoint of the airfoil) of the turbine.

See Tables 3, 4, and 5.

\section{Acknowledgment}

The support of General Electric Corporate Research and Development is acknowledged in this work.

\section{References}

[1] B. Lakshminarayana, Fluid Dynamics and Heat Transfer of Turbomachinery, Wiley-Interscience, New York, NY, USA, 1995.

[2] H. Cohen, C. F. C. Rogers, and H. I. H. Saravanamuttoo, Gas Turbine Theory, Longman, Essex, UK, 4th edition, 1996.

[3] M. B. Giles and M. Drela, "Two dimensional transonic aerodynamic design method," AIAA Journal, vol. 25, no. 9, pp. 1199-1206.

[4] M. Drela and M. B. Giles, "Viscous inviscid analysis of transonic and low reynolds number airfoils," AIAA Journal, vol. 25 , no. 10 , pp. 1347-1355.

[5] T. Dang, "Inverse method for turbomachine blades using shock capturing techniques," in Proceedings of the Joint Propulsion Conference and Exhibit, San Diego, Calif, USA, July 1995.

[6] O. Leonard and R. A. Van den Braembussche, "Design method for subsonic and transonic cascade with prescribed mach number distribution," Journal of Turbomachinery, vol. 114, pp. 553-560, 1992.

[7] S. Goel and S. H. Lamson, "Knowledge-based system for detailed blade design of turbines," in Proceedings of the Applications of Artificial Intelligence: Knowledge Based Systems in Aerospace and Industry, vol. 1994 of Proceedings of SPIE, Orlando, Fla, USA, 1995.

[8] S. Goel and S. H. Lamson, "Automating the design process for 3D turbine blades," in Proceeding of the International Mechanical Engineering Congress and Exposition, vol. 232, San Francisco, Calif, USA, November 1995.
[9] S. Goel, J. I. Cofer, and H. Singh, "Turbine airfoil design optimization," in Proceedings of the International Gas Turbine And Aeroengine Congress and Exposition, Birmingham, UK, June 1996.

[10] G. W. Burgreen and O. Baysal, "Three-dimensional aerodynamic shape optimization of wings using sensitivity analysis," in Proceedings of the AIAA 32nd Aerospace Sciences Meeting \& Exhibit, Reno, Nev, USA, 1994.

[11] A. Chattopadhyay, N. Pagaldipti, and K. T. Chang, "A design optimization procedure for efficient turbine airfoil design," Computers \& Mathematics with Applications, vol. 26, no. 4, pp. 21-31, 1993.

[12] D. H. Huddleston and C. W. Mastin, "Optimization methods applied to aerodynamic design problems in computational fluid dynamics," in Proceedings of the 7th International conference of Finite Element Methods in Fluid Flow, Huntsville, Ala, USA, 1989.

[13] A. Jameson, "Optimum aerodynamic design using control theory," Computational Fluid Dynamics Review, vol. 3, pp. 495-528, 1995.

[14] J. Reuther and A. Jameson, "Supersonic wing and wing body shape optimization using an adjoint formulation," in Proceedings of the ASME International Mechanical Engineering Congress and Exposition, San Francisco, Calif, USA, November 1995.

[15] H. Cabuk and V. Modi, "Optimum plane diffusers in laminar flow," Journal of Fluid Mechanics, vol. 237, pp. 373-393, 1992.

[16] J. C. Lewis and R. Agrawal, "Airfoil design via control theory using full potential and euler equations," in Proceedings of the ASME International Mechanical Engineering Congress and Exposition, vol. 232, San Francisco, Calif, USA, 1995.

[17] G. Farin, Curves and Surfaces for Computer Aided Geometric Design, Academic Press, San Diego, Calif, USA, 3rd edition, 1993.

[18] M. L. Shelton, B. A. Gregory, S. H. Lamson, H. L. Moses, R. L. Doughty, and T. Kiss, "Optimization of a transonic turbine airfoil using artificial intelligence, CFD, and cascade testing," in Proceedings of the ASME Gas Turbine Conference, vol. 93, Cincinnati, Ohio, USA, June 1993, 93-GT-161.

[19] R. R. Wysong, T. C. Prince, D. T. Lenahan, et al., "Turbine design system," Tech. Rep. TR 78 92, General Electric Company, Cincinnati, Ohio, USA, 1978.

[20] S. Goel and T. H. Kwon, "Geometric reasoning for automated design of an injection mold cooling system," in Proceedings of the IAPR International Workshop on Structural and Syntactic Pattern Recognition, Berne, Switzerland, August 1992.

[21] S. Goel, Machine learning paradigms in design optimization: applications in turbine aerodynamic design, Ph.D. thesis, Rensselaer Polytechnic Institute, New York, NY, USA, 1998.

[22] S. Goel and M. Embrechts, "Quantification of shape characteristics by using neural networks," in Intelligent Engineering Systems Through Artificial Neural Networks, Smart Engineering Systems: Neural Networks, Fuzzy Logic and Evolutionary Programming, C. H. Dagli, M. Akay, C. L. P. Chen, B. Fernandez, and J. Ghosh, Eds., vol. 6, 1996.

[23] E. O. Brigham, The Fast Fourier Transform, Prentice-Hall, Englewood Cliffs, NJ, USA, 1974. 

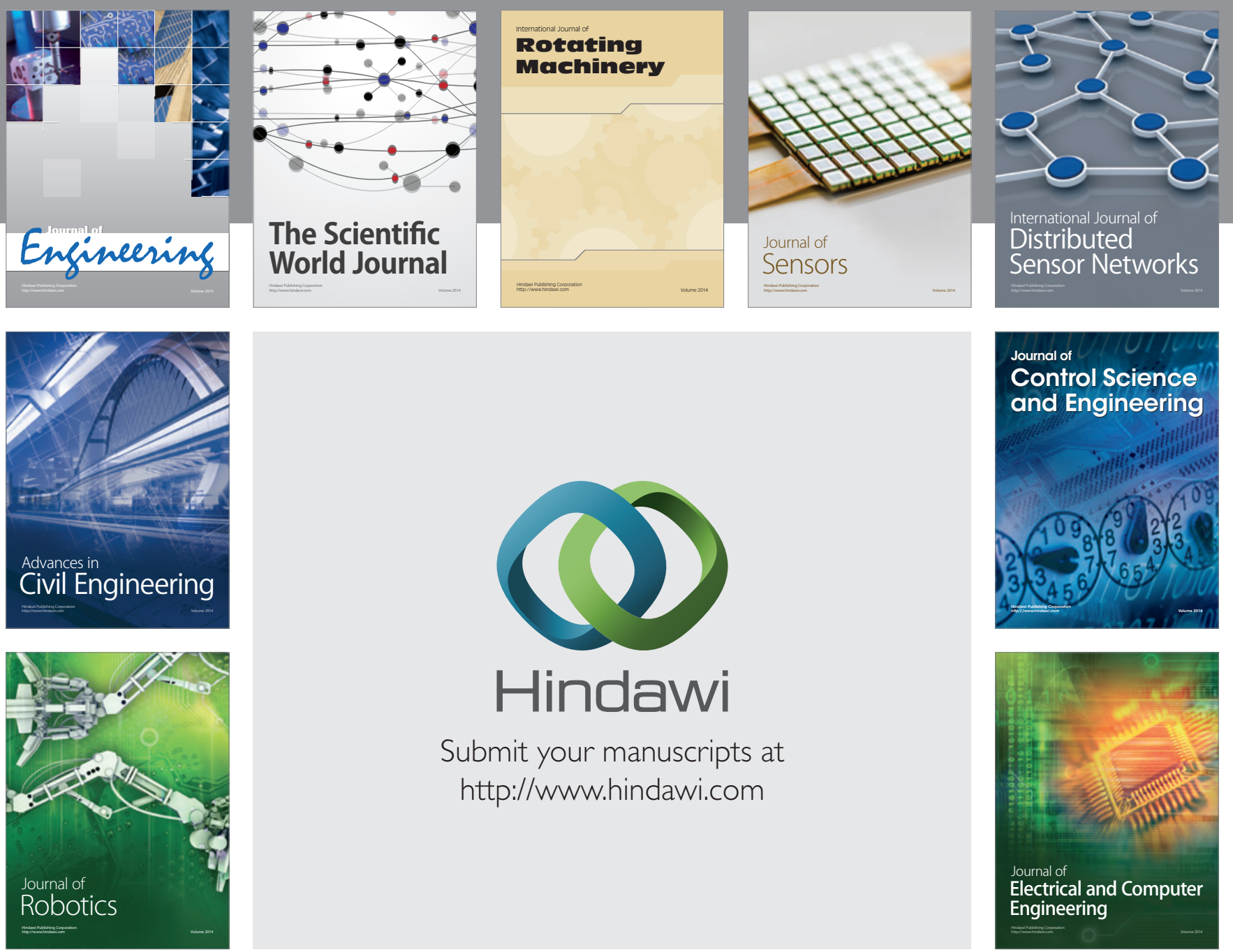

Submit your manuscripts at

http://www.hindawi.com
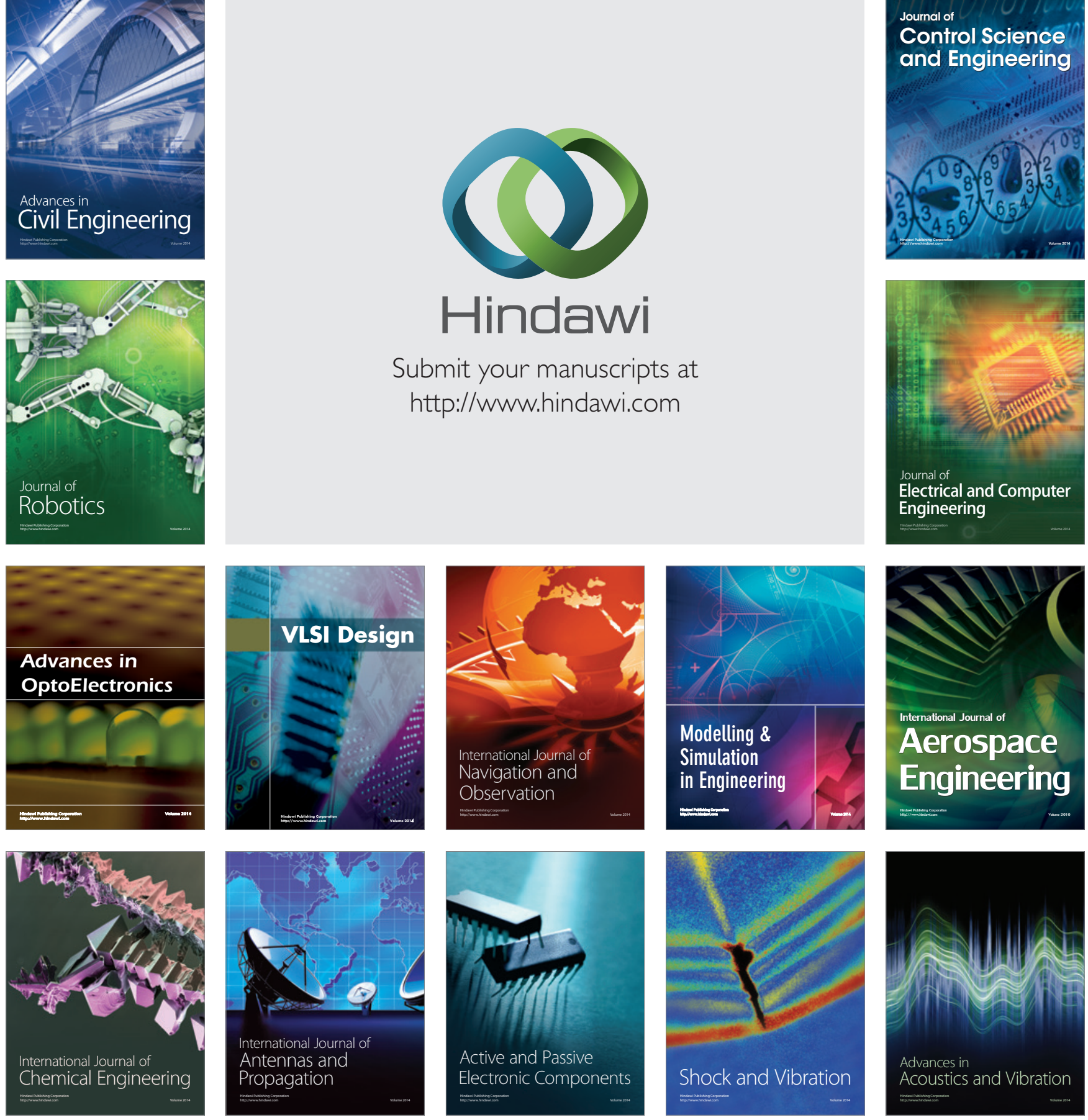